\title{
Effect of Shoulder Position on Scapular Muscle Activity during Scapular Protraction
}

\author{
Sung Joon Yun', Moon-Hwan Kim¹, Jong-Hyuck Weon² \\ 'Department of Rehabilitation Medicine, Wonju Severance Christian Hospital, Yonsei University, Wonju; ${ }^{2}$ Department of Physical Therapy, College of \\ Tourism \& Health Science, Joongbu University, Geumsan, Korea
}

Purpose: This study was to evaluate several tasks performed at a high intensity in terms of their ability to elicit EMG activity in the serratus anterior by comparing the EMG activities of the serratus anterior, upper trapezius, and lower trapezius muscles during six tasks combined shoulder flexion with rotation.

Methods: Fifteen healthy males were recruited to this study. Each subject was instructed to assume a sitting position without back support and asked to flex $\left(90^{\circ}\right.$ or $\left.120^{\circ}\right)$ the right shoulder and protract the scapula in the sagittal plane with maximal external rotation; to assume a neutral position; or to internally rotate the glenohumeral joint. The EMG data were collected from the serratus anterior (SA), upper trapezius (UT), and lower trapezius (LT) muscles were normalized to maximum voluntary isometric contraction. The UT/LT and UT/ SA muscle activity ratios in each task were assessed by calculating the surface EMG. Data were analyzed by two-way repeated-measures analysis of variance, with the level of significance set at $p<0.05$.

Results: The results of this study, shoulder flexion with external rotation resulted in low upper trapezius/serratus anterior and upper trapezius/lower trapezius ratios and a relatively high level of serratus anterior activation.

Conclusion: Shoulder flexion with external rotation used herein may be considered as important for clinical interventions aimed at selectively increasing SA strengthen and clinical selection of exercises for improving glenohumeral joint and scapulothoracic control.

Keywords: Protraction, Serratus anterior, Shoulder position, Strengthening

\section{INTRODUCTION}

The serratus anterior (SA) is unique among the scapulothoracic (ST) muscles in that it contributes to all aspects of the normal three-dimensional movement of the scapula on the thorax during elevation of the arm. ${ }^{1}$ The importance of the SA is evidenced by the presence of abnormal muscle activation in various shoulder pathologies, such as scapular dyskinematics and shoulder impingement syndrome.,2 Patients with shoulder impingement have increased electromyography (EMG) activity in the trapezius, but decreased EMG activity in the SA muscle during shoulder elevation in the scapular plane. ${ }^{1} \mathrm{~A}$ reduction in SA EMG activity may lead to compensatory overactivation of synergists of the upper trapezius (UT) muscle. ${ }^{1}$

Patients with an UT/SA imbalance also tend to have less scapular

Received May 19, 2020 Revised Jun 22, 2020

Accepted Jun 23, 2020

Corresponding author Jong-Hyuck Weon

E-mail jhweon@joongbu.ac.kr control and more scapular winging during shoulder flexion, as compared with abduction, because the trapezius is less able to compensate for the weak SA in flexion. ${ }^{3}$ For patients with an imbalance in SA to UT activation, an exercise with a low UT/SA ratio would be an important component of rehabilitation to allow selective SA strengthening and reduction of the imbalance. ${ }^{4}$ Proper balance of UT and SA activation is believed to reduce excess superior translation of the scapula, improve scapular posterior tipping, and maximize the available subacromial space beneath the acromion, thereby reducing rotator cuff impingement. Consequently, the SA is increasingly a focus of therapeutic exercises for the prevention and rehabilitation of shoulder dysfunction.

The SA is influenced by several factors, such as exercise, forward head and shoulder posture, shoulder flexion angle, hand grip

Copylight (C)2020 The Korean Society of Physical Therapy

This is an Open Access article distribute under the terms of the Creative Commons Attribution Non-commercial License (https:// creativecommons.org/license/by-nc/4.o.) which permits unrestricted non-commercial use, distribution, and reproduction in any medium, provided the original work is properly cited. 
strength, and hand external load. ${ }^{4-7}$ The restoration of scapulohumeral kinematics may require exercises to strengthen the SA and other scapular stabilizers. ${ }^{8}$ The selection of therapeutic exercises for enhancing SA activation has been based on EMG data. ${ }^{59-11}$ Increasing EMG activity in the SA, as the upper limb is actively flexed or abducted to the end of range of motion, causes maximum upward rotation of the scapula. ${ }^{9}$ Weon et al. ${ }^{8}$ reported on the importance of head position during exercise, and showed that maintaining a neutral head posture enhanced the SA activity. In addition, forearm rotation altered activation of the shoulder muscles. ${ }^{12}$ Resisted arm elevation above $120^{\circ}$ in various planes produced the maximum SA activity. ${ }^{6}$

Robert Lachaine et al. ${ }^{13}$ demonstrated that the upper arm rotation position affected the contributions of the SA and glenohumeral (GH) muscles. They also demonstrated that upper arm rotation affected the activity of the scapulohumeral muscles. However, no study has examined the effects of arm position on the SA. Several researchers reported EMG data for the SA and UT during various push-up exercises, although there have been no reports on the magnitude of SA, LT (lower trapezius), or UT activity, or the UT/SA and UT/LT ratios. ${ }^{3,5,6}$ Therefore, this study evaluated several tasks performed at a high intensity in terms of their ability to elicit EMG activity in the SA by comparing the EMG activities of the SA, UT, and LT muscles, and the UT/SA and UT/LT ratios, during combined shoulder flexion and GH rotation tasks.

\section{METHODS}

\section{Subjects}

The study enrolled 15 healthy males (mean age $=24.3 \pm 2.1$ years, height $=173.5 \pm 2.8 \mathrm{~cm}$, weight $=73.9 \pm 9.2 \mathrm{~kg}$ ). Subjects were excluded from the study if they had shoulder problems, such as tendonitis, adhesive capsulitis, instability, or impingement. All subjects were right hand dominant. All participants provided written informed consent before enrollment.

\section{Experimental methods}

The EMG data were collected from the SA, UT, and LT muscles using a TeleMyo 2400GT G2 data acquisition system (Noraxon, Scottsdale, AZ, USA). The analog signals were converted into digital signals, and the converted signals were processed for analysis using
MyoResearch software (MR-XP ver. 1.07, Noraxon). The sampling rate was set at $1,000 \mathrm{~Hz}$. A $10-450 \mathrm{~Hz}$ bandpass filter and $60 \mathrm{~Hz}$ notch filter were used to minimize electrical noise. The raw data were processed into root mean squares (RMS) with a $150 \mathrm{~ms}$ Window.

Before the surface electrodes were placed, the skin was shaved, abraded, and swabbed with alcohol-soaked cotton to minimize skin resistance. A small amount of electrode gel was applied to the silver chloride electrodes (DE-3.1 double differential electrode, Delsys, Boston, MA, USA), which were then applied to the skin. Pairs of surface electrodes were positioned bilaterally and parallel to the muscle fibers, at an interelectrode distance of $2 \mathrm{~cm}$ from the following muscles: the SA (in the anterior midaxillary region, over the fifth or sixth rib, anterior to the latissimus dorsi and placed vertically); the UT (midway between the acromion and cervical spine); and the LT (at an oblique vertical angle with one electrode superior, and one inferior, to a point $5 \mathrm{~cm}$ inferomedial from the root of the spine of the scapula). ${ }^{14}$

For normalization, the mean RMS of a 5 seconds maximal voluntary isometric contraction (MVIC) was measured three times for the SA, UT, and LT muscles, with subjects in the standard midaxillary position as demonstrated by Kendall et al. ${ }^{7}$ for measuring the MVIC. For the inferomedial SA, the subjects were supine, with the scapula abducted, projecting the upper extremity anteriorly (upward from the table), with resistance provided by a tester (downward pressure on the extremity to the scapula in the direction of scapular adduction); for the UT, the subjects were in a sitting position, with the acromial ends of the clavicle and scapula elevated and the neck extended posterolaterally, bringing the occiput toward the elevated shoulder with the face turned in the opposite direction. Resistance at the shoulder and head was provided by a tester pushing in the shoulder depression with the head flexed anterolaterally. For the LT, the subjects were prone, with the scapula adducted and depressed, lateral rotation of the inferior angle, and resistance applied at the forearm in a downward direction, i.e., toward the table. The UT/LT and UT/SA muscle activity ratios in each task were assessed by calculating the EMG ratio. To calculate the ratios, the normalized value of the UT was divided by the normalized value of the LT and SA, respectively. The ratio was considered low if it was less than 0.3 , while ratios close to or greater than 1 indicated similar activation by the two muscles or greater UT activation compared with 
that of the other muscle. ${ }^{15}$

\section{Procedures}

Each subject was instructed to assume a sitting position and maintain an erect posture without back support. While sitting on the chair, the subjects were asked to protract the scapula and flex $\left(90^{\circ}\right.$ or $120^{\circ}$ ) the right shoulder with maximal external rotation (SFER); to assume a neutral position (SFNP); or to internally rotate (SFIR) in the sagittal plane. The postures were performed in a random order. EMG data were acquired from the SA, UT, and LT muscles using surface electrodes. Before testing, all subjects were familiarized with shoulder flexion, and with scapular protraction in the sagittal plane via use of a $2 \mathrm{~kg}$ dumbbell in the SFER, SFNP, and SFIR postures, exerting maximal effort while sitting with an erect posture without back support. During the familiarization process, the subjects were instructed to maintain shoulder flexion of the right upper limb at $90^{\circ}$ or $120^{\circ}$ and to perform scapular protraction. To minimize muscle fatigue, after the familiarization period a 10 minutes rest period was provided before data collection. An inclinometer was used to determine when the shoulder was at $90^{\circ}$ or $120^{\circ}$ of flexion. A horizontal bar was placed at this height and subjects were given feedback when instructed to flex the right shoulder, and to protract the scapula using a $2 \mathrm{~kg}$ dumbbell without elbow flexion (with the subject in a sitting position to prevent trunk lateral bending, rotation and thoracic extension). The subjects were requested to hold the task postures for 5 seconds, with each posture being repeated three times. To minimize muscle fatigue, a 3 minutes rest period was provided between tests. EMG signals were collected during each 5 seconds isometric contraction.

\section{Statistical Analysis}

PASW for Windows software (ver. 18.0, SPSS Inc., Chicago, IL, USA) was used to analyze the data, which are expressed as means \pm SD. Two-way repeated-measures analysis of variance (ANOVA) was used, with the level of significance set at $\mathrm{p}<0.05$, to determine the effect of the shoulder flexion angle and rotation on the SA, UT, and LT muscle activities, and on the muscle activity ratios (UT/SA and UT/LT). Interactions between factors were also evaluated; if a significant interaction between the shoulder flexion angle $\left(90^{\circ}\right.$ and $120^{\circ}$ ) and rotation (external rotation, neutral position, and internal rotation) was observed, the simple effect was compared using the Bonferroni correction.

\section{RESULTS}

Table 1 presents the mean $( \pm \mathrm{SD})$ normalized EMG activation data for the SA, UT, and LT muscles (Table 1).

\section{Serratus anterior}

There was a significant difference was found for $\mathrm{GH}$ joint rotation $(\mathrm{p}<0.05)$. The SA activity was significantly greater in SFER than in SFNP. There was no significant main effect of shoulder flexion angle ( $\mathrm{p}>0.05)$, and interaction of shoulder flexion angle and GH rotation of SA muscle was not significant.

Table 1. Mean ( \pm SD) EMG activity of the serratus anterior, upper trapezius, and lower trapezius expressed as a percentage of \%MVIC for 6 tasks

\begin{tabular}{|c|c|c|c|c|c|c|c|}
\hline \multirow{2}{*}{ Muscle } & \multirow{2}{*}{$\begin{array}{l}\text { Shoulder } \\
\text { flexion }\end{array}$} & \multicolumn{3}{|c|}{ Glenohumeral joint rotation } & & \multirow{2}{*}{$\mathrm{F}$} & \multirow{2}{*}{$p$} \\
\hline & & SFER & SFNP & SFIR & & & \\
\hline \multirow[t]{3}{*}{ SA } & $90^{\circ}$ & $55.16 \pm 17.50$ & $45.92 \pm 14.38$ & $52.48 \pm 17.33$ & angle & 0.035 & 0.853 \\
\hline & & & & & rotation & 7.602 & $0.004^{*}$ \\
\hline & $120^{\circ}$ & $66.17 \pm 27.43$ & $57.64 \pm 25.23$ & $58.45 \pm 23.03$ & angle*rotation & 2.281 & 0.98 \\
\hline \multirow[t]{3}{*}{ UT } & $90^{\circ}$ & $8.27 \pm 7.11$ & $8.77 \pm 8.16$ & $17.57 \pm 20.38$ & angle & 11.291 & $0.003^{*}$ \\
\hline & & & & & rotation & 28.305 & $<0.001^{*}$ \\
\hline & $120^{\circ}$ & $25.85 \pm 19.55$ & $27.97 \pm 23.85$ & $38.38 \pm 29.94$ & angle $*$ rotation & 0.557 & 0.464 \\
\hline \multirow[t]{3}{*}{ LT } & $90^{\circ}$ & $7.59 \pm 6.08$ & $7.59 \pm 5.92$ & $13.92 \pm 10.70$ & angle & 9.23 & $0.006^{*}$ \\
\hline & & & & & rotation & 1.263 & 0.274 \\
\hline & $120^{\circ}$ & $8.07 \pm 6.56$ & $8.35 \pm 7.11$ & $16.31 \pm 14.25$ & angle*rotation & 1.047 & 0.318 \\
\hline
\end{tabular}

SA: serratus anterior muscle, UT: upper trapezius muscle, LT: lower trapezius, SFER: shoulder flexion with external rotation, SFNP: shoulder flexion with neutral position, SFIR: shoulder flexion with internal rotation.

${ }^{*} p<0.05$. 

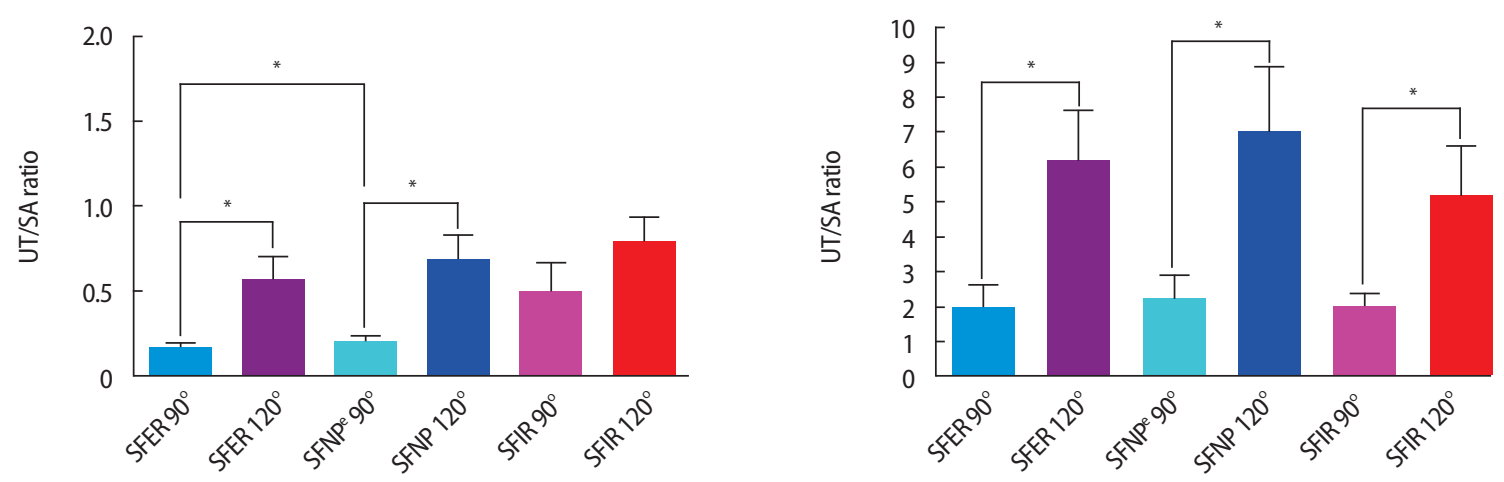

Figure 1. Bar graphs show the ratio of UT/SA and UT/LT during 6 tasks SA: serratus anterior muscle, UT: upper trapezius muscle, LT: lower trapezius, SFER: shoulder flexion with external rotation, SFNP: shoulder flexion with neutral position, SFIR: shoulder flexion with internal rotation. ${ }^{*} \mathrm{p}<0.05$.

\section{Upper trapezius}

There were significant main effects of both shoulder flexion angle and $\mathrm{GH}$ rotation $(\mathrm{p}<0.05, \mathrm{p}<0.001)$. The UT muscle activity was significantly greater shoulder flexion of $120^{\circ}$ than $90^{\circ}$. Moreover, the UT activity was greater in SFIR than in SFER or SFNP $(\mathrm{p}=0.009$ and $\mathrm{p}=0.002$, respectively). No significant interaction was observed between shoulder flexion angle and rotation.

\section{Lower trapezius}

There was a significant main effect was found for shoulder flexion angle $(\mathrm{p}<0.05)$. The LT activity was significant greater flexion $120^{\circ}$ than $90^{\circ}$. There was no significant difference GH rotation, but SFIR show greater LT activity than in SFER or SFNP. There was no significant interaction of angle and GH rotation was observed.

\section{Ratio of UT to SA, UT to LT}

There was a significant main effect was observed for shoulder flexion angle (UT/SA: $\mathrm{F}=10.779, \mathrm{p}<0.05 ; \mathrm{UT} / \mathrm{LT}: \mathrm{F}=8.590, \mathrm{p}=0.008)$ and $\mathrm{GH}$ rotation $(\mathrm{UT} / \mathrm{SA}: \mathrm{F}=6.295, \mathrm{p}=0.018)$. The UT/SA and UT/ LT ratios were significantly greater with shoulder flexion of $120^{\circ}$ versus $90^{\circ}$. In addition, UT/SA ratio was significant lower in SFER than SFNP $(\mathrm{p}<0.05)$. There was no significant interaction of shoulder flexion angle and GH rotation. (Figure 1).

\section{DISCUSSION}

The surface EMG signal provides a good representation of the activity of an entire muscle and is more reliable than analyzing muscle activity with intra-muscular electrodes. ${ }^{16}$ The current study investi- gated the EMG activity of the SA, UT, and LT muscles and compared the UT/SA and UT/LT ratios between postures demanding different degrees of shoulder flexion and GH rotation. The SA activity was significantly greater in SFER, while the UT activity increased significantly in SFIR. The UT and LT activities increased significantly with shoulder flexion of $120^{\circ}$. The UT/SA and UT/LT ratios differed significantly by shoulder flexion angle. SFER $90^{\circ}$ showed the lowest UT/SA ratio among the tasks, while shoulder flexion at $90^{\circ}$ had a significantly lower UT/LT ratio than flexion at $120^{\circ}$.

Selective activation of the SA without marked activation of the UT may improve the relative strength of the SA and the balance of activation between these muscles in patients with shoulder dysfunction. ${ }^{1,2}$ We found that SFER induced the greatest SA activation. These findings of greater SA activity during protraction exercises can be explained in two ways. First, because of the leftward shift in the length-tension curve, the SFER position induces upward scapular rotation, which may result in greater SA activity (because the muscle is maintained in a shortened position). Second, in the testing position, maximal SFER can lead to increased activity of the external rotators of the $\mathrm{GH}$ joint, which may result in more motion at the ST joint compared with the GH joint. The SA needs to contract for upward rotation of the ST joint, while maintaining maximal SFER, regardless of the shoulder flexion angle.

However, there was no significant effect of shoulder flexion angle. Previous studies demonstrated that the SA muscle affects depending on the angle of the shoulder flexion, especially, a maximum EMG amplitude at $120^{\circ}$ of shoulder flexion, but the results of this study did not accord with those findings. ${ }^{5,7}$ This was thought to be 
due condition of the GH joint (maximal external rotation, internal rotation, and neutral position). Excessive activation of the GH joint external rotator muscles induced downward scapular rotation during shoulder flexion. This suggests that the SA does in order to prevent the scapular downward rotation of maximal SFER with scapular protraction, thus increasing the activity of the SA. Therefore, the change in the GH rotation might have affected the SA activity, because of maintaining of upward scapular rotation.

Individuals with shoulder pain show increased UT activation and reduced SA activation, as well as increased superior translation of the scapula. ${ }^{1}$ In addition, excess activation of the UT, combined with poor control of the LT and SA, may play a major role in abnormal scapular motion and the development of shoulder pathology.,17 In our study, SFIR $120^{\circ}$ resulted in significantly greater EMG activity of the UT. These findings could be associated with the testing position, i.e., upward scapular rotation with shoulder internal rotation. Maintaining the testing position not only keeps the UT muscle in a shortened position, but also affects its level of activation. During an empty-can exercise, Robert Lachaine et al. ${ }^{13}$ reported that an increased contribution from the ST was associated with a compensatory pattern that limited the contribution of the GH. Therefore, previous findings of position-induced changes in kinematics, muscle activation patterns, and the length-tension relationship are consistent with our findings. ${ }^{13}$

The LT performs a stabilizing function, in which the fibers operate at a constant length to maintain the axis of rotation about the GH joint, rather than functioning as a torque generator. ${ }^{17}$ We found that LT muscle produced significant greater EMG amplitude at $120^{\circ}$ of shoulder flexion than at $90^{\circ}$, but no significant main effect between GH rotations. However, SFIR produced greater activation of the LT than the other postures. Robert Lachaine et al. ${ }^{13}$ demonstrated that SFIR acted to increase the ST contribution, and that subtle differences in arm position affect the activation of the LT (because of the leftward shift in the length-tension curve). The LT appears to be essential for maintaining proper postural alignment and GH function.18 Thus, our findings support the results of Robert Lachaine et al. ${ }^{13}$

In our study, the UT/SA ratio was significantly lower for SFER $90^{\circ}$ than other postures, indicating greater relative activity of the SA. The UT/LT ratio was no significant effect of GH rotation, but UT/LT ratio was lower in SFER, SFNP, and SFIR $90^{\circ}$. These findings are in good agreement with the result of a previous study, and low UT/SA and UT/LT ratios would be an important component of an exercise program to allow selective SA and LT muscle strengthening. ${ }^{1}$ Cool et al. ${ }^{17}$ reported that excess activation of the UT muscle may cause abnormal scapular and shoulder motion. Therefore, reducing the activation of the UT may be important in shoulder rehabilitation. We found that $\mathrm{SFER}^{\circ}$ had significantly lower UT/SA and UT/LT ratios than the other postures. These findings suggest that activation of the SA, UT, and LT muscles is dependent on shoulder flexion or rotation of the GH joint, which has important implications for rehabilitation.

A few limitations to this study should be noted. First, each participant had a different range of maximum shoulder rotation and different scapulohumeral rhythms during shoulder flexion, which we could not fully control for. Second, muscles other than the SA, UT, and LT, which can contribute to GH and ST control, were not considered in this investigation. Future research needs to investigate whether activation of the supraspinatus, pectoralis major, infraspinatus, and deltoid muscles contributes to GH and ST control in accordance with the arm position.

Shoulder flexion with external rotation resulted in low UT/SA and UT/LT ratios and a relatively high level of SA activation. The postures used herein may be considered as important for clinical interventions aimed at selectively increasing SA strengthen. In addition, both maximal activation of the SA, and lower UT/SA and UT/ LT ratios, should affect the clinical selection of exercises for improving GH joint and ST control.

\section{REFERENCES}

1. Ludewig PM, Cook TM. Alterations in shoulder kinematics and associated muscle activity in people with symptoms of shoulder impingement. Phys Ther. 2000;80(3):276-91.

2. Sahrmann S. Movement system impairment syndromes of the extremities, cervical and thoracic spines. Elsevier/Mosby, 2011:21-8.

3. Inman VT, Abbott LC. Observations of the function of the shoulder joint. 1944. Clin orthop Relat Res. 1996;330:3-12.

4. Shim YH, Nam KS, Park JW. Effect of glenohumeral joint rotation on serratus anterior, pectoralis major and upper trapezius EMG activity during push-up plus exercise. J Kor Phys Ther. 2016;28(2):106-11.

5. Ekstrom RA, Donatelli RA, Soderberg GL. Surface electromyographic analysis of exercises for the trapezius and serratus anterior muscles. J Orthop Sports Phys Ther. 2003;33(5):247-58.

6. Ekstrom RA, Bifulco KM, Lopau CJ et al. Comparing the function of the 
upper and lower parts of the serratus anterior muscle using surface electromyography. J Orthop Sports Phys Ther. 2004;34(5):235-43.

7. Kendall FP, McCreary EK, Provance PG et al. Muscles: Testing and function with posture and pain. 5th ed. Baltimore, MD, Williams \& Wilkins, 2005:321-39.

8. Weon JH, Oh JS, Cynn HS et al. Influence of forward head posture on scapular upward rotators during isometric shoulder flexion. J Bodyw Mov Ther. 2010;14(4):367-74.

9. Ludewig PM, Cook TM, Nawoczenski DA. Three-dimensional scapular orientation and muscle activity at selected positions of humeral elevation. J Orthop Sports Phys Ther. 1996;24(2):57-65.

10. Nam KS, Kim JW, Park JW. Comparative analysis of the serratus anterior muscle activity when right and left knee lift during quadruped position on push up plus. J Kor Phys Ther. 2020;32(1):58-63.

11. Kim TH, Kim SY, Park HK. Effect of shoulder protraction exercise on the supine position for selective activation of the serratus anterior according to weight in winging scapula. J Kor Phys Ther. 2019;31(4):199203.

12. Chen HM, Leung CT. The effect on forearm and shoulder muscle activity in using different slanted computer mice. Clin Biomech. 2007;22(5):518-23. 13. Robert Lachaine X, Allard P, Gobout V et al. Shoulder coordination during full-can and empty-can rehabilitation exercises. J Athl Train. 2015;50(11):1117-25.

14. Criswell E. Cram's introduction to surface electromyography. Jones \& Bartlett Publishers, 2010:289-97.

15. Martins J, Tucci HT, Andrade R et al. Electromyographic amplitude ratio of serratus anterior and upper trapezius muscles during modified pushups and bench press exercises. J Strength Cond Res. 2008;22(2):477-84.

16. Kadaba M, Wootten M, Gainey J et al. Repeatability of phasic muscle activity: Performance of surface and intramuscular wire electrodes in gait analysis. J Orthop Res. 1985;3(3):350-9.

17. Cools AM, Witvrouw EE, De Clercq GA et al. Scapular muscle recruitment pattern: electromyographic response of the trapezius muscle to sudden shoulder movement before and after a fatiguing exercise. J Orthop Sports Phys Ther. 2002;32(5):221-9.

18. Arlotta M, LoVasco G, McLean L. Selective recruitment of the lower fibers of the trapezius muscle. J Electromyogr Kinesiol. 2011;21(3):40310. 